ORIGINAL ARTICLE

\title{
The relationships between dynamic balance and sprint, flexibility, strength, jump in junior soccer players
}

\author{
Alpaslan Kartal ${ }^{\mathrm{ABCDE}}$ \\ Yozgat Bozok University Physical Education and Sport Department, Turkey
}

Authors' Contribution: A - Study design; B - Data collection; C - Statistical analysis; D - Manuscript Preparation; E - Funds Collection.

\begin{abstract}
Purpose:

The present study aimed to investigate the relationships between sprint, flexibility, upper extremity strength and Star Excursion Balance Test performances in soccer players aged 12-14 years.

Material: $\quad$ Eighteen junior soccer players (mean age, $9.78 \pm 1.6$ years; height, $139.5 \pm 11.0 \mathrm{~cm}$; weight, $34.3 \pm 9.9$ $\mathrm{kg}$; BMI, 17.3 \pm 2.9 ; leg length; $63.7 \pm 5.9 \mathrm{~cm}$ ) participated in this study voluntarily. They were training 2 or 3 times a week with the addition of one match per month and none of them had reported injuries or diseases related to sports. After measuring the anthropometrics of the subjects, they were familiarized with the test procedures. Measurements included handgrip strength (HD), $30 \mathrm{sc}$. sit-ups (SU), standing long jump (SLJ), Countermovement jump (CMJ) and 20m-Sprint tests as independent variables, and Star Excursion Balance Test (SEBT) as dependent variables. Then, statistical analysis was applied to the data transferred to SPSS 24.0 Packet program.

Results: $\quad$ The inconsistent correlations between variables of SEBT and selected parameters were found. The strongest significant relationships of the variables of SEBT with selected variables were observed between anterolateral $(A L)$ and $S U(r=646, p<0.05)$, and between posteromedial (PM) and Sprint $(r=-650)$. No significant correlations were observed between variables of dynamic balance and CMJ, SLJ, Relative Handgrip Strength (RHS).

Conclusions: $\quad$ As a result, strength and power performances may not necessarily be related to impaired balance directly in young soccer men. Moreover, low back flexibility may have negative or positive influences on dynamic balance.

Keywords: $\quad$ strength, flexibility, soccer, sprint, anthropometry, balance
\end{abstract}

\section{Introduction}

It has been well documented that physical fitness components that may affect overall athletic performance have relationships with each other. Particularly, muscular strength as a major fitness component is related to other components such as speed, power, endurance and balance. Previous studies on this topic have revealed that the more an athlete can increase their strength or power, the greater their endurance performance, balance and sprint ability will occur. Likewise, dynamic balance ability, which is an important component for overall athletic performance for many sports, may be affected by several performances such as strength and flexibility [2]. On the other hand, the effect size of these relationships varies according to such as age, gender, sports, specific training and performances $[3,4]$.

It is clear that relationships between physical fitness components can be critical for athletes and coaches in terms of strength/conditioning and sports medicine. With respect to sports medicine, Caspersen, Powell, \& Christenson, which is one of the first studies investigating the relationship between balance and muscle strength/power, revealed that skill-related components of physical fitness are not independent with each other [5]. Furthermore, this association is important for the identification of atrisk individuals because deficits in these neuromuscular

(c) Alpaslan Kartal, 2020

doi:10.15561/26649837.2020.0602 components are associated with an increased risk of sustaining injuries and falls [6]. When considered the fact that dynamic balance is also linked to cognitive functions [7] as muscular strength may [8], the studies investigating the relationship between strength and balance should be interpreted comprehensively. Muehlbauer et al. have also deduced that "these relationships are important from two perspectives: (a) testing and identifying at-risk individuals, and (b) developing and implementing individually tailored injury and fall-prevention programs" [9]. Although the importance of this issue is clear in terms of medicine (general health and sports) and athletic performance, there are limited studies that have been reported until today. Moreover, it is hard to compare the findings of previous studies due to they have methodological differences in terms of testing models and populations. With respect to measurements, while some studies have used isokinetic tests for strength measurements, some with performing functional strength tests. Furthermore, their dynamic balance testing models were different. For instance, Star Excursion Balance Test (SEBT) which is most commonly utilized as a postural control testing is a dynamic test that requires strength, flexibility, and proprioception [6]. On the contrary flexibility and strength are not necessary for other balance tests (static balance tests, postural sway or other reactive balance tests). In addition to measurement differences, population differences have an impact on the interrelationships between athletic performances. It is 
previously reported that the factors of age and maturation affect athletic performances and injuries [10].

In addition, sports have different physical and physiological demands, so players' athletic performances (strength, endurance, power, speed, agility, balance, etc.) vary by sports type. Since several team sports like soccer demands both aerobic and anaerobic conditioning variables such as pace, agility, balance, muscular strength and endurance [6] and interpreting the relationships between fitness components in those sports should also be elaborated. Previous studies have shown that small-tomedium correlations $(\mathrm{r}<.40)$ between strength, flexibility and balance $[6,9]$. A recently conducted systematic review on the relationship between dynamic balance, strength and power detected that there are small-sized correlation coefficients between these parameters in children, adolescents, and young, middle-aged, and old adults across the lifespan but age/maturation may have an impact [9]. Hammami, Chaouachi, Makhlouf, Granacher, \& Behm have also stated that knowledge about the relationship between balance and strength, power might provide greater insight into the way maturity modifies the effectiveness of different training regimes (e.g., balance and strength training). Considering the literature, the present study aimed to investigate the relationships between sprint, flexibility, upper extremity strength and Star Excursion Balance Test performances in soccer players aged $12-14$ years.

\section{Materials and Methods}

\section{Participants}

Eighteen junior soccer players who had been playing for a farm team of a first division team in Turkey (mean age, $9.78 \pm 1.6$ years; height, $139.5 \pm 11.0 \mathrm{~cm}$; weight, $34.3 \pm 9.9 \mathrm{~kg}$; BMI, 17.3 \pm 2.9 ; leg length; $63.7 \pm 5.9 \mathrm{~cm}$ ) participated in this study voluntarily. They were training 2 or 3 times a week with the addition of one match per month (there were no official tournaments for adolescent players) and none of them had reported injuries or diseases related to sports. The inclusion criteria were (1) have more than a 6-month detraining period (2) at least 1 year of systematic soccer training with a minimum of three training sessions per week (i.e.,90 min per session). (3) have suffered any musculoskeletal, neurological and orthopedic disorder that might have affected their ability to perform strength, power and balance tests.

After measuring the anthropometrics of the subjects, they were familiarized with the test procedures.

\section{Research Design.}

Measurements included handgrip strength (HD), 30 sc. sit-ups (SU), standing long jump (SLJ), Countermovement jump (CMJ) and 20m-Sprint tests as independent variables, and Star Excursion Balance Test (SEBT) as dependent variables. As body weight is a determinant factor in strength parameters [12] relative values were computed separately for handgrip strength but not for sit-up test (BW has no or even negative impact on Sit-up performance).

Dynamic Balance.
SEBT was to evaluate the dynamic balance abilities of the subject. The subject performed to reach 8 different directions; anterolateral (AL), anterior (ANT), anteromedial (AM), medial (MD), posteromedial (PM), posterior (PO), posterolateral (PL), and lateral (LAT). It is previously demonstrated that reach distances of SEBT test should be normalized to leg length to obtain more accurate dynamic postural control scores [13]; therefore, normalized values for each subject were computed. All subjects wore athletic shoes during the performance of the test.

\section{Jump}

Jump performances were tested using by the Optojump (Optojump, Microgate, Bolzano, Italy) and assessed by vertical countermovement jump (CMJ) with arm swing during the execution of the jump (i.e., hands were free to move) distances. The subject had performed three times, with 45 seconds of passive recovery and the highest scores (in centimetres) were recorded [14]. Standing long jump (SLJ) is an effective and practical test for predictive muscular strength and power [11] and so this test was also measured.

\section{Sprint}

This distance was chosen because it is valid and highly reliable in physically active individuals (correlation coefficient of 0.91 between test and retest) [15]. Tests were conducted using photoelectric cells (Witty, Microgate, Bolzano, Italy) and performed on a synthetic grass pitch. Participants were permitted three trials, and the best performance was recorded.

Strength

The muscular strength of the upper extremity was determined by using a handgrip dynamometer (Takei, Tokyo) and applying a 30-sec sit-up test (SU). To obtain SU values, the participants performed as many repetitions as possible within 30 seconds, as previously described [16].

\section{Flexibility}

The sit-and-reach test (SAR), which is a common form of low back and hamstring flexibility with an accuracy of $0.5 \mathrm{~cm}$ [17], was employed.

Statistical Analysis.

SPSS Version 24.0 was used for statistical analysis. After checking the assumptions (absence of outliers and normality), Pearson analysis was performed to obtain correlation coefficients and the relationships were interpreted by Cohen's standards.

\section{Results}

Means and SDs are presented for all the variables in Table 1 and Table 2 respectively.

The highest mean reach distances were obtained in $\mathrm{MD}$, and the lowest mean reach distances were in LAT direction for both raw and normalized values.

The inconsistent correlations between variables of SEBT and selected parameters were found. There were medium positive or negative correlations between SAR and the SEBT variables of ANT, AL, PO and PM, and positive medium correlation dependences with $\mathrm{HS}$ and $\mathrm{AL}$ 
Table 1. Descriptive statistics for dependent variables

\begin{tabular}{lll}
\hline Reaching Directions & Raw Scores $\mathbf{( c m )}$ & $\begin{array}{l}\text { Normalized } \\
\text { (\% of leg length) }\end{array}$ \\
\hline AM & $57.3 \pm 6.4$ & $90.0 \pm 6.1$ \\
ANT & $57.8 \pm 9.6$ & $90.5 \pm 11.2$ \\
AL & $54.2 \pm 8.1$ & $84.9 \pm 8.1$ \\
LAT & $53.7 \pm 9.3$ & $84.3 \pm 12.0$ \\
PL & $57.4 \pm 10.8$ & $90.3 \pm 15.8$ \\
PO & $60.2 \pm 11.4$ & $94.1 \pm 14.2$ \\
PM & $61.1 \pm 10.2$ & $95.4 \pm 10.1$ \\
MD & $63.7 \pm 9.8$ & $99.7 \pm 10.9$ \\
\hline
\end{tabular}

NOTE: $\mathrm{AL}=$ anterolateral, $\mathrm{ANT}=$ anterior, $\mathrm{AM}=$ anteromedial, $\mathrm{MD}=$ medial, $\mathrm{PM}=$ posteromedial, $\mathrm{PO}=$ posterior, $\mathrm{PL}=$ posterolateral and $\mathrm{LAT}=$ lateral

Table 2. Descriptive statistics for independent variables

\begin{tabular}{lllllll}
\hline \multirow{2}{*}{$\begin{array}{l}\text { Sprint } \\
(\mathbf{2 0 m})\end{array}$} & Flexibility & Jumps & \multicolumn{3}{c}{ Strength } \\
\cline { 2 - 7 } & SAR $(\mathbf{c m})$ & CMJ $(\mathbf{c m})$ & SLJ $(\mathbf{c m})$ & HS $(\mathbf{k g})$ & RHS(BW/kg) & SU \\
\hline $41.2 \pm 3.6$ & $20.2 \pm 3.4$ & $23.4 \pm 4.7$ & $141.2 \pm 22.3$ & $18.1 \pm 3.8$ & $0.55 \pm 0.10$ & $20.9 \pm 3.6$ \\
\hline
\end{tabular}

NOTE: $\mathrm{SAR}=\mathrm{Sit}$ and reach, $\mathrm{CMJ}=$ Countermovement jump, $\mathrm{SLJ}=$ Standing Long Jump, HS= Handgrip Strength, RHS= Relative Handgrip Strength, SU= Sit-ups

Table 3. The correlations between dynamic balance and selected athletic performances

\begin{tabular}{llllllll}
\hline $\begin{array}{l}\text { SEBT } \\
\text { Variables }\end{array}$ & SAR $(\mathbf{c m})$ & CMJ (cm) & SLJ $(\mathbf{c m})$ & HS (kg) & RHS(BW/kg) & SU (30sc) & Sprint(20m) \\
\hline AM & -168 & 296 & 080 & 025 & -371 & 129 & 245 \\
ANT & $477^{*}$ & 436 & 372 & 093 & -379 & 237 & -409 \\
AL & $482^{*}$ & 373 & 373 & $508 *$ & 045 & $646 *$ & -313 \\
LAT & -187 & 210 & 019 & 251 & 117 & 290 & -266 \\
PL & -181 & 099 & 035 & 132 & 006 & 235 & -155 \\
PO & $-445^{*}$ & 209 & 102 & 354 & 067 & 334 & -254 \\
PM & $-539 *$ & 252 & 318 & $552^{*}$ & 082 & 368 & $-650^{*}$ \\
MD & 319 & 290 & 204 & 438 & 125 & 265 & 318 \\
\hline
\end{tabular}

NOTE: * $\mathrm{P}<0.05 \mathrm{AL}=$ anterolateral, $\mathrm{ANT}=$ anterior, $\mathrm{AM}=$ anteromedial, $\mathrm{MD}=$ medial, $\mathrm{PM}=$ posteromedial, $\mathrm{PO}=$ posterior, $\mathrm{PL}=$ posterolateral and $\mathrm{LAT}=$ lateral, $\mathrm{SAR}=\mathrm{Sit}$ and reach, $\mathrm{CMJ}=$ Countermovement jump, $\mathrm{SLJ}=$ Standing Long Jump, HS= Handgrip Strength, RHS= Relative Handgrip Strength, SU= Sit-ups

and $\mathrm{PM}(\mathrm{P}<0.05)$. The strongest significant relationships of the variables of SEBT with selected variables were observed between AL and SU $(r=646, \mathrm{p}<0.05)$, and between PM and Sprint $(r=-650)$. However, no other SEBT variables were correlated with SU or Sprint. No significant correlations were observed between variables of dynamic balance and CMJ, SLJ, RHS.

\section{Discussion}

The primary finding of this study is that inconsistent correlations between variables of SEBT and other selected athletic performance variables. The most surprising results were that while some relationships between SEBT variables and SAR were found positively significant, others were found negatively significant. It is difficult to explain these inverse relationships; however, it is likely that while forward reaching skills may have positive, and backward have negative associations with lower extremity flexibility. Athletes with optimum flexibility can be more capable of performing dynamic balance ability, but those with high or low flexibility may be susceptible to perform postural control ability. Since the SEBT requires strength, flexibility, neuromuscular control, core stability, ROM, balance, and proprioception [18], 1 component can cause any disruption in this test. Overmoyer GV and Reiser RF suggested in their study that when used with recreationally active adults, the $\mathrm{Y}$ Balance Test may be helpful to establish lower-extremity flexibility deficits and flexibility asymmetries in the ankle and hip regions but may need to be used in conjunction with additional 
tests to understand a broader picture [2]. Another possible reason for these inverse relationships may be attributed to factors related to soccer-specific. Soccer players are required to perform repetitive and explosive unilateral movements such as sudden acceleration and deceleration tasks, rapid changes of direction, kicking, jumping and landing [19] and these performances may bring about enhancement of anterior reach performances of postural stability, and flexibility.

The present study also reported significant relationships between some variables of strength and dynamic balance. There were positive and medium-sized correlations of AL with SU and HS, and of PM with HS. These findings were consistent with previous studies that found associations between strength and dynamic balance performances; however, is that correlation coefficients were different, with some studies reporting small-sized, some with medium-sized and some with strongly positive correlations [9]. Still, it should also be considered that much of these studies have used lower extremity strength measurements, unlike the current study. Another critical point is that those correlation coefficients are different in previous studies.
Jumping and sprinting are high-intensity performances, and also known as power performances, so it was expected that these variables would show similar correlations with dynamic balance variables. Apart from a significant relationship between $20 \mathrm{~m}$ sprint and PM, there were no significant relationships between these parameters and any SEBT variables. Izquierdo, Aguado, Gonzalez, Lopez, \& Häkkinenstated that dynamic balance is not affected by power or strength components [20]. In that study, similar jump tests (SLJ and CMJ) and different dynamic balance tests were carried out. Our findings indicate that dynamic balance and jump performances are independent of each.

\section{Conclusions}

In conclusion, strength and power performances may not necessarily be related to impaired balance directly in young soccer men. And, low back flexibility may have negative or positive influences on dynamic balance.

\section{Conflicts of Interest}

The author declare no conflict of interest.

\section{References}

1. Wisloeff U, Helgerud JAN, Hoff JAN. Strength and endurance of elite soccer players. Med. Sci. Sport. Exerc. 1998;30(3):462-467. https://doi.org/10.1097/00005768-199803000-00019

2. Overmoyer G V, Reiser RF. Relationships between lowerextremity flexibility, asymmetries, and the $\mathrm{Y}$ balance test. J. Strength Cond. Res. 2015;29(5):1240-1247. https://doi.org/10.1519/JSC.0000000000000693

3. Popovic S, Akpinar S, Jaksic D, et al. Comparative study of anthropometric measurement and body composition between elite soccer and basketball players. Int. J. Morphol. 2013;31(2):461-467. https://doi.org/10.4067/S0717-95022013000200016

4. Ünlü G, Çevikol C, Melekoğlu T. Comparison of the Effects of Eccentric, Concentric, and EccentricConcentric Isotonic Resistance Training at Two Velocities on Strength and Muscle Hypertrophy. Journal of Strength and Conditioning Research, 2020;34:337-44. https://doi.org/10.1519/JSC.0000000000003086

5. Caspersen CJ, Powell KE, Christenson GM. Physical activity, exercise, and physical fitness: definitions and distinctions for health-related research. Public Health Rep. 1985;100(2):126.

6. Butler RJ, Southers C, Gorman PP, Kiesel KB, Plisky PJ. Differences in soccer players' dynamic balance across levels of competition. J. Athl. Train. 2012;47(6):616-620. https://doi.org/10.4085/1062-6050-47.5.14

7. TaekemaDG,LingCHY,KurrleSE, etal.Temporal relationship between handgrip strength and cognitive performance in oldest old people. Age Ageing. 2012;41(4):506-512. https://doi.org/10.1093/ageing/afs013

8. Leandri M, Campbell J, Molfetta L, Barbera C, Tabaton M. Relationship between balance and cognitive performance in older people. J. Alzheimer's Dis. 2015;45(3):705-707. https://doi.org/10.3233/JAD-142883

9. Muehlbauer T, Gollhofer A, Granacher U. Associations between measures of balance and lower-extremity muscle strength/power in healthy individuals across the lifespan: a systematic review and metaanalysis. Sport. Med. 2015;45(12):1671-1692. https://doi.org/10.1007/s40279-015-0390-z

10.Williams HG, Pfeiffer KA, O'neill JR, et al. Motor skill performance and physical activity in preschool children. Obesity. 2008;16(6):1421-1426. https://doi.org/10.1038/oby.2008.214

11.Hammami R, Chaouachi A, Makhlouf I, Granacher U, Behm DG. Associations between balance and muscle strength, power performance in male youth athletes of different maturity status. Pediatr. Exerc. Sci. 2016;28(4):521-534. https://doi.org/10.1123/pes.2015-0231

12.Isık A, Unlu G, Gozubuyuk OB, Aslanyurek T, Bereceli $C$. The relationship between previous lower extremity injury, body weight and bilateral eccentric hamstring strength imbalance in young soccer players. Montenegrin J. Sport. Sci. Med. 2018;7(2). https://doi.org/10.26773/mjssm.180904

13.Gribble PA, Hertel J. Considerations for normalizing measures of the Star Excursion Balance Test. Meas. Phys. Educ. Exerc. Sci. 2003;7(2):89-100. https://doi.org/10.1207/S15327841MPEE0702_3

14.Glatthorn JF, Gouge S, Nussbaumer S, et al. Validity and reliabilityofOptojumpphotoelectriccellsforestimatingvertical jump height. J. Strength Cond. Res. 2011;25(2):556-560. https://doi.org/10.1519/JSC.0b013e3181ccb18d

15.Delextrat A, Cohen D. Strength, power, speed, and agility of women basketball players according to playing position. J. Strength Cond. Res. 2009;23(7):1974-1981. https://doi.org/10.1519/JSC.0b013e3181b86a7e

16.Johnson BL, Nelson JK. Practical measurements for evaluation in physical education. 1969.

17.Opstoel K, Pion J, Elferink-Gemser $M$, et al. Anthropometric characteristics, physical fitness and motor coordination of 9 to 11 year old children participating in a wide range of sports. PLoS One. 2015;10(5):e0126282. https://doi.org/10.1371/journal.pone.0126282

18.Gribble PA, Hertel J, Plisky P. Using the Star Excursion 
Balance Test to assess dynamic postural-control deficits and outcomes in lower extremity injury: a literature and systematic review. J. Athl. Train. 2012;47(3):339-357. https://doi.org/10.4085/1062-6050-47.3.08

19.López-Valenciano A, Ayala F, Croix MDS, Barbado D, VeraGarcia FJ. Different neuromuscular parameters influence dynamic balance in male and female football players. Knee
Surgery, Sport. Traumatol. Arthrosc. 2019;27(3):962-970. https://doi.org/10.1007/s00167-018-5088-y

20.Izquierdo M, Aguado X, Gonzalez R, Lopez JL, Häkkinen K. Maximal and explosive force production capacity and balance performance in men of different ages. Eur. J. Appl. Physiol. Occup. Physiol. 1999;79(3):260-267. https://doi.org/10.1007/s004210050504

\section{Information about the author:}

Alpaslan Kartal; https://orcid.org/0000-0003-1567-6276; akartal6161@hotmail.com; Yozgat Bozok University Physical Education and Sport Department; Atatürk Yolu 7. Km 66900 Yozgat, Turkey.

Cite this article as:

Kartal A. The relationships between dynamic balance and sprint, flexibility, strength, jump in junior soccer players. Pedagogy of Physical Culture and Sports, 2020;24(6):285-289.

https://doi.org/10.15561/26649837.2020.0602

This is an Open Access article distributed under the terms of the Creative Commons Attribution License, which permits unrestricted use, distribution, and reproduction in any medium, provided the original work is properly cited (http://creativecommons.org/licenses/by/4.0/deed.en).

Received: 10.05.2020

Accepted: 24.06.2020; Published: 30.12.2020 